\title{
Identifying emotion subtypes from individualized emotion space
}

\author{
Seth M. Levine ${ }^{\bowtie}$, Aino L. I. Alahäivälä , Anja Wackerle , Rainer Rupprecht , and Jens V. Schwarzbach ${ }^{凶}$ \\ Department of Psychiatry and Psychotherapy, University of Regensburg, Regensburg 93053, Germany
}

\begin{abstract}
Many investigations into emotion processing contend that emotions can be reduced to a set of lower dimensions (e.g., valence and arousal). Additionally, emotion dysregulation is associated with numerous psychiatric disorders, whose treatment(s) may require inspiration from personalized medicine. To translate emotion research to the clinical domain, one may therefore need to investigate at the individual level, employing datadriven methods and forgoing classical assumptions regarding emotions. To this end, we explored the relative structure of emotion information resulting from 85 participants organizing emotionally-charged images following their own emotional responses to the pictures. Using cluster analyses and multidimensional scaling, we investigated the underlying composition of individuals' emotion spaces. Hierarchical clustering revealed five subtypes that reflect differing layouts of the emotion space; multidimensional scaling of each subtype's representative emotion space demonstrated that, although valence explained the primary organization of all emotion spaces, arousal as a secondary explanatory variable played a reduced role differentially for the subtypes, suggesting intrinsic differences in emotion information processing. Such data-driven methods yield new, unbiased ways of studying emotions and may reveal limitations of classic models or idiosyncrasies of individuals, which can inform future neuroimaging research and offer new approaches for studying emotions and emotion dysfunctions in psychiatric disorders.
\end{abstract}

Correspondence: seth.levine@ukr.de, jens.schwarzbach@ukr.de

\section{Introduction}

For millennia the nature of human emotions has been a topic of intense debate. Despite recent advances in the domain of neuroscience, the manner in which the brain represents emotions remains unknown. Although there is consensus that a one-to-one mapping of emotions to brain areas does not exist ${ }^{1-3}$, aspects of the circumplex model ${ }^{4}$, which explains emotion states as a combination of valence (i.e., pleasant/unpleasant) and arousal (i.e., stimulating/nonstimulating), have pervaded the psychology domain ${ }^{5,6}$ and subsequently motivated a variety of recent neuroimaging studies $^{7-12}$ that sought the neural correlates of emotions.

In addition to the general interest of understanding the nature of human emotions, such knowledge is crucial for helping those afflicted with emotion dysregulation ${ }^{13}$, which has been linked to a variety of psychiatric disorders ${ }^{14-19}$. The push toward personalized medicine ${ }^{20}$, breaking away from the rigidity of traditional theories of emotions ${ }^{3}$, and improving computational and translational methods for psychiatry $^{21-23}$ led us to opt for a data-driven method to study emotions. We adapted the multi-arrangement method $^{24}$ and inverse multidimensional scaling ${ }^{25}$, often employed within the domain of visual cognitive neuroscience ${ }^{26-29}$, to emotionally-charged stimuli in order to uncover participants' subjective structure of such emotion information. Having participants freely organize images according to their emotional similarity in a continuous space yielded a representation of the individual "emotion space" within the cognitive architecture, which allowed us to further investigate its underlying structure using data-driven cluster analyses.

Here we present results from this behavioral experiment, which demonstrate the presence of emotion subtypes, whose emotion spaces are explained differentially by classical emotion models. This data-driven approach can be used as a tool to inform cognitive science and computational psychiatry, allowing researchers to determine, for example, how and potentially why emotion dysregulations arise in certain psychiatric disorders, and whether subtyping of emotions can improve the sensitivity of neuroimaging analyses or alter future diagnostic and treatment methods.

\section{Methods}

\section{Participants}

Eighty-five participants (31 males, 54 females; age range = $20-33$ yrs) were recruited from the local community via information posters. Participants neither had a current diagnosis of neuropsychiatric disorders nor were taking any psychotropic medication. All participants provided written informed consent before taking part in the study and were monetarily compensated for their time after completing the experiment. All experimental procedures complied with the Declaration of Helsinki and were approved by the local ethics committee at the University of Regensburg.

\section{Apparatus}

The experiment was conducted on a 27" Apple iMac using MATLAB R2015b (The Mathworks, Natick, MA, USA). The MATLAB code used to run the experiment was adapted from that described by Kriegeskorte and Mur ${ }^{25}$. All analyses were also carried out in MATLAB.

\section{Stimuli}

Images used in the experiment were obtained from the International Affective Picture System (IAPS) database ${ }^{30}$. The IAPS database contains images with standardized valence and arousal ratings that can be roughly divided into nine categories: animals, people, nature, food, household items, erotic 


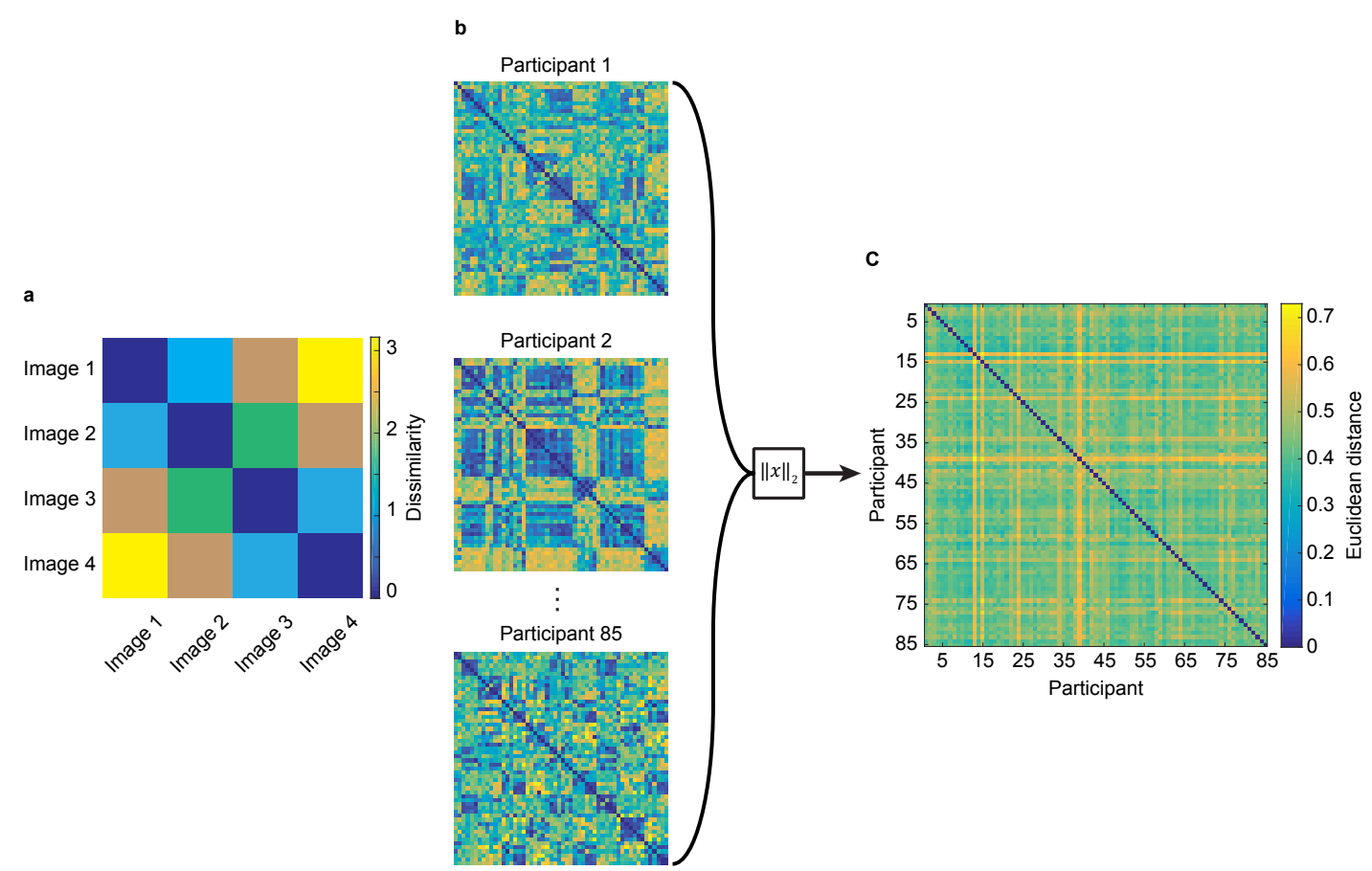

Fig. 1. After participants organized stimuli on the computer screen according to the emotional similarity of the stimuli, we computed the distance between each pair of stimuli and compiled the pairwise distances between them into a dissimilarity matrix. An example scenario in which only four images appeared in the experiment would yield (a) a $4 \times 4$ dissimilarity matrix, which is symmetric across the main diagonal. In our experiment, 85 participants organized 54 stimuli (see Supplementary table 1 for stimulus information), resulting in (b) 85 participant-specific $54 \times 54$ dissimilarity matrices, each reflecting the underlying high-dimensional structure of a given participant's emotion space. We computed the Euclidean distance (symbolically denoted here by the L2 norm) between all pairs of matrices, resulting in (c) an $85 \times 85$ matrix, which reflects the dissimilarity between all participants. We then applied further cluster analyses (see Figure 2) to this matrix in order to reveal underlying structure that explains similarities and differences between participants in terms of their emotion spaces.

images, accidents, violence, and war. We pseudo-randomly chose six images per category for the experiment, yielding a stimulus set comprising 54 images. If the computer algorithm chose two images that were very visually similar (e.g., two pictures of a dog in a similar position, or two pictures of knives on a table), we manually selected a replacement image with the same (or very similar) valence and arousal values from that category (see Supplementary table 1 for the corresponding image ID's and their respective valence and arousal ratings). Creating the stimulus set was ultimately a trade-off between improved sampling of the emotion space and total experiment duration, as the distance between each pair of images must be computed; the total number of pairwise distances is given by the binomial coefficient $\left(\begin{array}{l}n \\ 2\end{array}\right)$, which in our case was $1431(n=54)$, resulting in a total experiment duration of, on average, 1.12 hours for a given participant.

\section{Trial protocol}

The experiment followed the protocol laid out by Kriegeskorte and $\mathrm{Mur}^{25}$, in which participants arrange images on a twodimensional circular "arena" on a computer screen according to some organizing principle. We asked participants to arrange pictures according to how they felt when they viewed each image. That is, images evoking similar emotions should be placed closer together while images evoking dissimilar emotions should be placed further apart; thus the distance between images reflects their relative emotional dissimilarity for the participant (see Figure 1a for the concept). Partic- ipants were informed that the ultimate structure is entirely individualized and that there was no "correct answer" for this experiment.

No more than ten images were shown during a given trial to improve visibility of the pictures (i.e., fitting 54 pictures on the screen at the same time would render them all so small that their content would become unrecognizable). When the participant finished organizing the images, the next trial began with a new set of images, for which the inverse multidimensional scaling algorithm had the least evidence regarding their relative distances among all 54 images. Thus, some of the images may have been present in two successive trials. After evidence for all 1431 pairwise dissimilarities was obtained, inverse multidimensional scaling transformed the two-dimensional distances on the computer screen into a 54 $\times 54$ dissimilarity matrix (DSM; Figure $1 b)^{25}$, which represents the high-dimensional dissimilarity structure of the item arrangements.

\section{Data analysis}

\section{Hierarchical clustering}

In order to uncover whether emotion subtypes existed in our sample, we first computed the pairwise Euclidean distance between the lower-triangle (because the matrix is symmetric across the main diagonal) of participants' DSMs, yielding an $85 \times 85$ DSM reflecting the dissimilarity between each participant to all other participants (Figure $1 \mathrm{~b}$ and 1c). We 


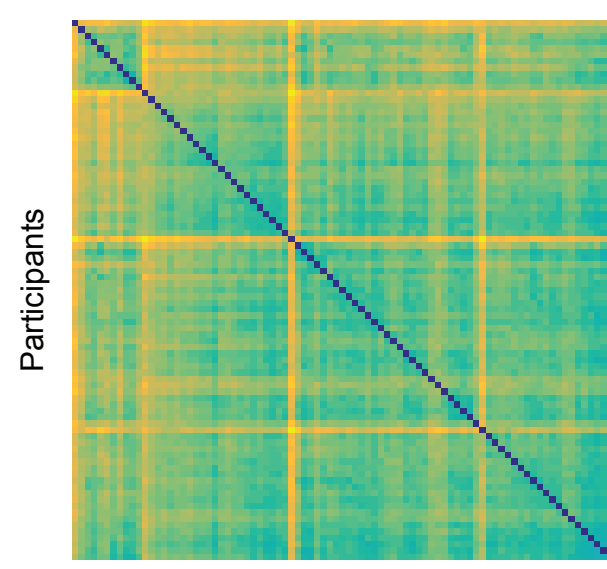

Participants
2 clusters

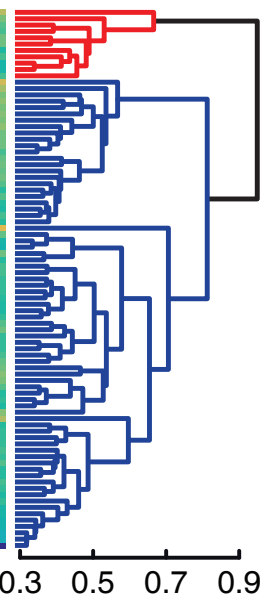

Euclidean distance
5 clusters

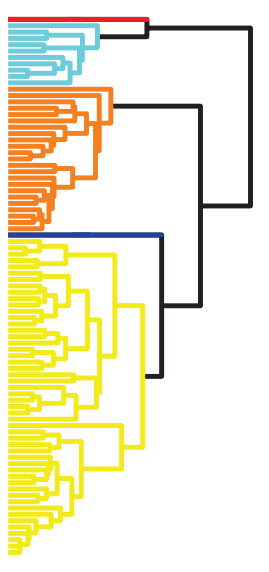

8 clusters

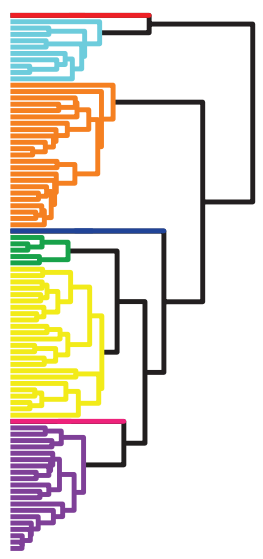

C

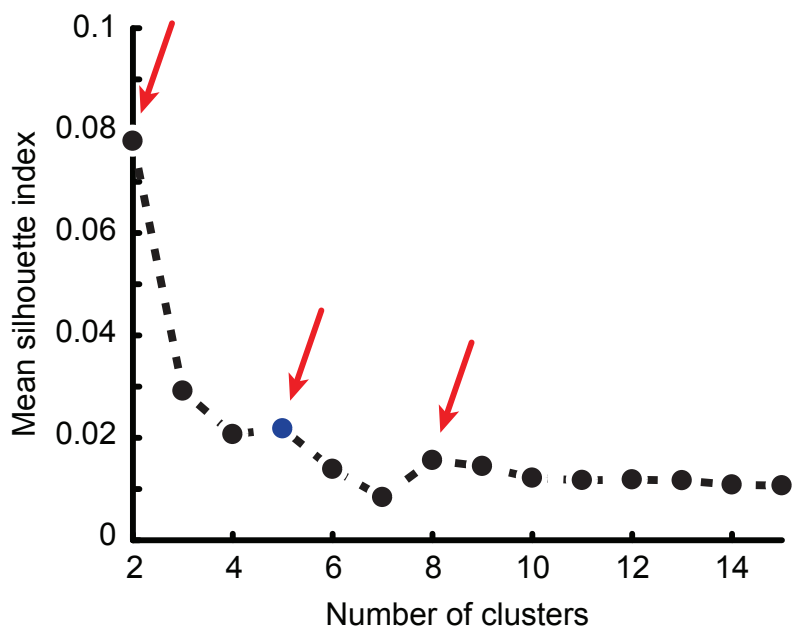

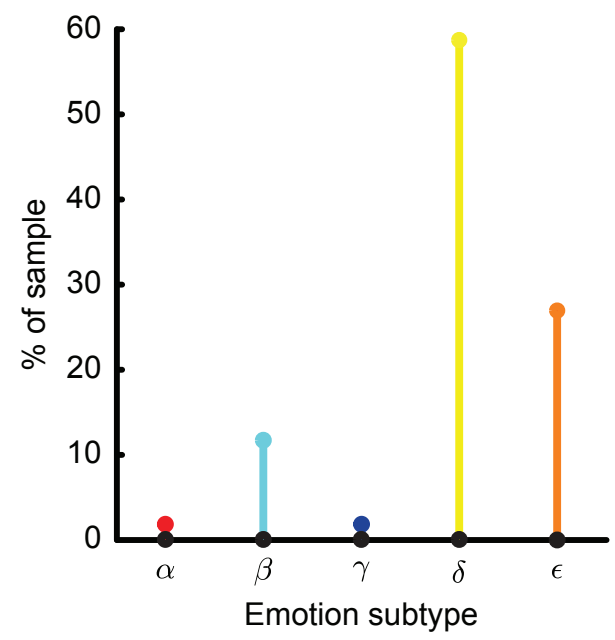

Fig. 2. (a) The participant-wise dissimilarity matrix reorganized according to the results from (b) the hierarchical clustering analysis, which revealed that participants (each leaf of the dendrogram) can be parsed into (left) two, (middle) five, or (right) eight groups (or emotion subtypes), represented by the different colors in the dendrogram. These clustering solutions were validated by (c) the silhouette index ${ }^{32}$, a measure of intra- vs. inter-cluster variance. Each peak in the silhouette profile denotes a level of "stability" that arises from the corresponding clustering solution. The two-, five-, and eight-cluster solutions (denoted by the red arrows) correspond to the global maximum, the first peak, and the second peak of the silhouette profile, respectively. The blue circle indicates the clustering solution that we used in further analyses. (d) A probability mass function depicting the proportion of the sample that each of the subtypes (designated as alpha through epsilon) from the five-cluster solution comprised.

then applied an agglomerative hierarchical clustering analysis to this DSM using Ward's minimum variance linkage criterion $^{31}$, to obtain a dendrogram that depicts the underlying structure of the high-dimensional DSM (Figure 2a and 2b).

\section{Cluster validation}

To identify sub-groups of participants with similar emotion spaces, we sought stable clusters in the hierarchical structure by iteratively cutting the dendrogram at different heights to create multiple clustering solutions and calculated the silhouette index ${ }^{32}$ for each solution, which computes the intracluster distance with respect to the inter-cluster distance of any given item's nearest neighboring cluster. Clustering solutions that yield higher silhouette indices are considered stable states and thus more "natural" than those that yield lower silhouette indices. As the expected value of the silhouette index approaches zero with more clusters present in the data, we took the first silhouette peak following a decrease as indicative of a clustering solution that yields unexpected, and therefore meaningful, stability in the system (Figure $2 \mathrm{c}$ and $2 \mathrm{~b}$ middle dendrogram). These clusters can represent emotion subtypes within the dendrogram generated from the subjectto-subject DSM.

\section{Resampling procedure}

To determine whether the clustering solutions we obtained were stable and not merely a fluke dependent on our sample size, we performed a resampling procedure, in which we recomputed the cluster validation on an iteratively increas- 


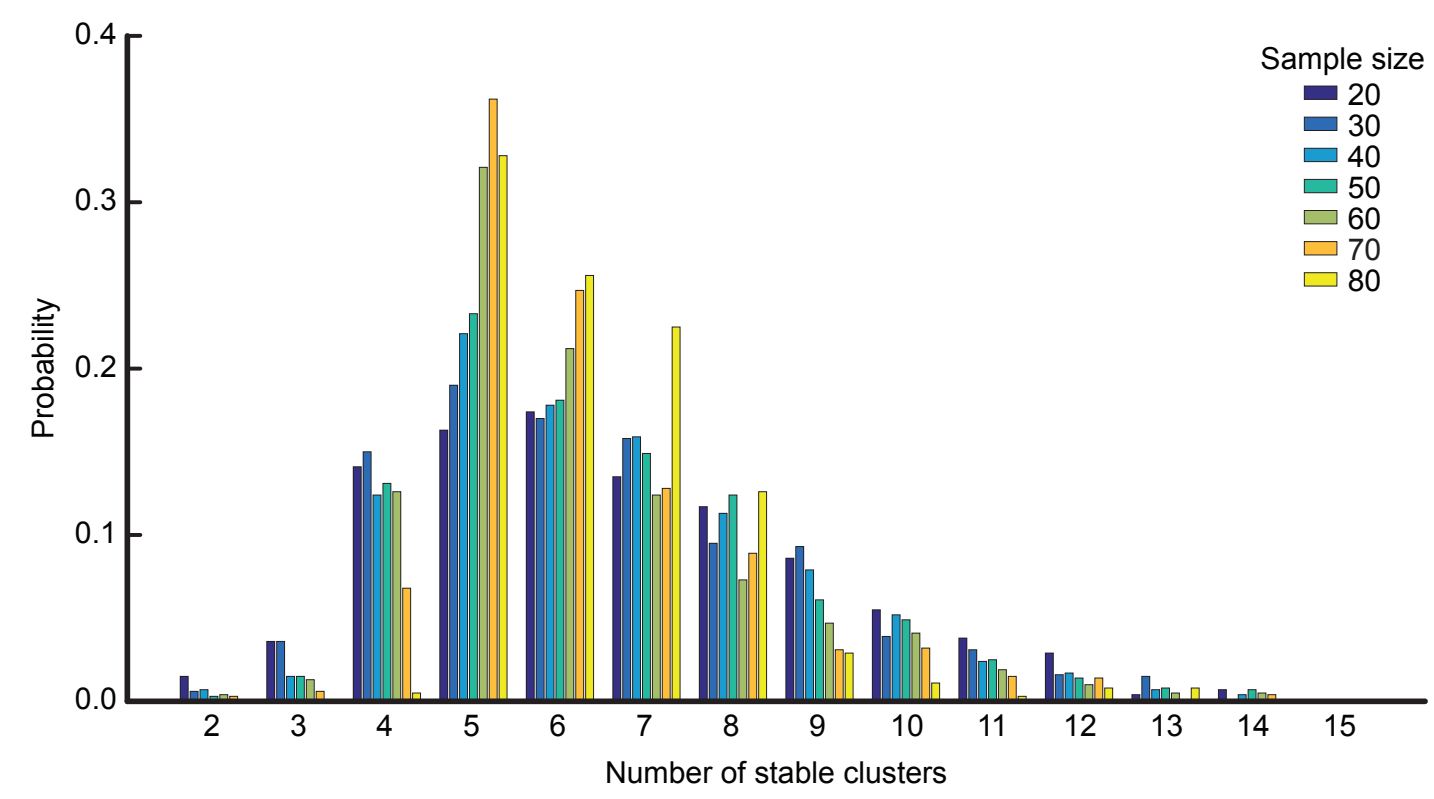

Fig. 3. Probability mass functions (PMFs) generated through a resampling procedure in which subsets of different sizes ( $n=20$ through 80 , in steps of 10 ) were sampled, without replacement, from the full sample of 85 participants. For each subset of size $\mathrm{n}$, we repeated the process of randomly sampling $\mathrm{n}$ dissimilarity matrices, performing hierarchical clustering, and calculating the first peak of the respective silhouette profile 1000 times resulting in seven PMFs, which reflect the probability of a given clustering solution as a function of the sample size. As the sample size increased, so did the probability of the five-clustering solution being the function's maximum. This procedure validated the five-cluster solution we found with the full sample of 85 participants.

ing subset of participants. Beginning with a random subset of 20 participants, we clustered the data, calculated the silhouette index, and stored the clustering solution that corresponded to the silhouette's first peak using the previously defined methods. This procedure was repeated 1000 times, without replacement, yielding a distribution of 1000 clustering solutions for unique subsets of 20 participants. We then repeated this resampling procedure for subsets of size 30,40 , $50,60,70$, and 80 , which ultimately yielded seven probability mass functions (PMFs) describing the probability of a given clustering solution as a function of the sample size. This process allowed us to determine that the results we obtained with 85 participants were justified, given the maxima of the PMFs (Figure 3). Although our maximum permutation space was $\left(\begin{array}{l}85 \\ 40\end{array}\right)$, which is on the order of 2.9 septillion, 1000 permutations, when taken at random, have been shown to represent the sampling space well ${ }^{33}$.

\section{Valence/arousal assessment}

To evaluate the ability of the valence-arousal model to describe the emotion space of each subtype, we averaged together all DSMs from the participants of a given subtype, resulting in five subtype-representative DSMs, and performed a non-metric multidimensional scaling, using Kruskal's stress1 criterion $^{34}$, on each subtype's representative DSM. Each DSM was reduced to four dimensions, as this was the minimum the number of dimensions required to bring the stress value for all five DSM's below $0.2^{34}$ (a stress value of 0 denotes no information lost during dimensionality reduction, i.e., a perfect transformation). We then correlated the organization of the stimuli along each dimension with the stimuli's valence and arousal scores. By computing the partial correlation (Pearson's r), we were able to assess the association of valence while accounting for the effect of arousal, and vice versa. P-values for correlation coefficients were calculated through a Student's t transformation. Statistical thresholding was set at $\mathrm{p}<0.05$ (two-tailed), with a False Discovery Rate (FDR) ${ }^{35}$ correction to account for the 40 tests (five subtypes $\times$ two explanatory variables $\times$ four dimensions) we performed.

\section{Data availability}

The datasets generated and/or analyzed during the current study are available from the corresponding author upon reasonable request.

\section{Results}

\section{Hierarchical clustering reveals five subtypes}

Computing the Euclidean distance between each pair of individual DSMs (Figure 1b) yielded a subject-to-subject DSM (Figure 1c), whose underlying structure was uncovered using hierarchical clustering (Figure $2 \mathrm{a}$ and $2 \mathrm{~b}$ ). Cluster validation using the silhouette index ${ }^{32}$ provided evidence for twocluster, five-cluster, and eight-cluster solutions (Figure 2c). For simplicity, here we focused on the five-cluster solution (see Yeo et al. ${ }^{36}$ for a similar concept), as it was neither the coarsest nor the finest "clustering resolution" (and thus the middle-ground) generated by our sample. One can consider the five clusters as emotion-subtypes, each constituting a different percentage of the sample: $\alpha=1 \%, \beta=12 \%, \gamma=1 \%, \delta$ $=59 \%, \epsilon=27 \%$ (Figure $2 \mathrm{~d}$ ), and not explainable by a simple male/female distinction $\left(\chi_{(4)}^{2}=5.19, \mathrm{p}<0.27\right)$. It should be noted that subtypes $\alpha$ and $\gamma$ are each composed of a single participant. 


\section{Resampling validates five subtypes}

Simulating smaller sample sizes through resampling without replacement demonstrated that the expected clustering solution using the first peak of the silhouette approaches five clusters as the sample size increases (Figure 3). Although the maximum of the 20-subject PMF was the 6-cluster solution at $17.4 \%$, the maxima for all subsequent PMFs (from 30 to 80 ) were the 5-cluster solution at $19 \%, 22.1 \%, 23.3 \%, 32.1 \%$, $36.2 \%$, and $32.8 \%$, respectively. This trend reinforces the idea that the five subtypes we identified from the 85 participants represent a stable solution and not a coincidence driven by a sample of insufficient size.

\section{Valence-arousal dimensions apply differentially to emotion subtypes}

Using non-metric multidimensional scaling, we reduced all subtype-representative DSMs to four dimensions (stress values: $\alpha=0.187, \beta=0.058, \gamma=0.085, \delta=0.056, \epsilon=0.114$; Figure 4). Computing partial correlations between the valence/arousal values with the organization of stimulus images along each dimension revealed that valence very strongly explained the layout of the first dimension for all five subtypes' emotion spaces $\left(\alpha: \mathrm{r}=-0.73, \mathrm{p}=7.7 \times 10^{-10} ; \beta\right.$ : $\mathrm{r}=-$ $0.90, \mathrm{p}=5.1 \times 10^{-20} ; \gamma: \mathrm{r}=0.72, \mathrm{p}=1.6 \times 10^{-9} ; \delta: \mathrm{r}$ $\left.=-0.948, \mathrm{p}=4.9 \times 10^{-25} ; \epsilon: \mathrm{r}=-0.92, \mathrm{p}=8.4 \times 10^{-23}\right)$, while arousal only did for subtype epsilon $(\epsilon: \mathrm{r}=0.49, \mathrm{p}=$ $\left.2.0 \times 10^{-4}\right)$ but not for the other subtypes $(\alpha: \mathrm{r}=0.37, \mathrm{p}=$ $6.2 \times 10^{-3} ; \beta: \mathrm{r}=0.32, \mathrm{p}=0.02, \gamma: \mathrm{r}=-0.03, \mathrm{p}=0.84 ; \delta$ : $\left.\mathrm{r}=0.37, \mathrm{p}=6.2 \times 10^{-3}\right)$. Alternatively, valence did not define the organization of the second dimension of the emotion spaces $(\alpha: \mathrm{r}=0.10, \mathrm{p}=0.49 ; \beta: \mathrm{r}=-0.01, \mathrm{p}=0.92, \gamma: \mathrm{r}=$ $0.03, \mathrm{p}=0.81 ; \delta: \mathrm{r}=0.24, \mathrm{p}=0.08 ; \epsilon: \mathrm{r}=0.12, \mathrm{p}=0.39$ ), whereas arousal did, but only for subtype delta $(\delta: \mathrm{r}=0.51$, $\left.\mathrm{p}=8.9 \times 10^{-5}\right)$ and not for the other subtypes $(\alpha: \mathrm{r}=0.17$, $\mathrm{p}=0.22 ; \beta: \mathrm{r}=0.16, \mathrm{p}=0.26 ; \gamma: \mathrm{r}=-0.31, \mathrm{p}=0.02 ; \epsilon: \mathrm{r}$ $=0.23, p=0.10)$. Interestingly, although valence and arousal played mostly no role in organizing the third and fourth dimensions of each subtype's emotion space $(\min (\mathrm{p})>0.025)$, arousal did correlate with the layout of the third dimension of subtype epsilon $\left(\epsilon: \mathrm{r}=-0.43, \mathrm{p}=1.4 \times 10^{-3}\right)$. For visualizations of the dimensionality-reduction and valence/arousal correlations with each dimension, see Figures 4 and 5, respectively; descriptive statistics for all four dimensions can be found in Supplementary table 2 and Supplementary table 3. Statistical thresholds were FDR-corrected $35(\mathrm{q}=0.05, \mathrm{p}$ $<1.4 \times 10^{-3}$ ).

\section{Discussion}

In this experiment, we adapted a behavioral method to the domain of emotion research and, using connectivity-based datadriven analyses, identified emotion subtypes distinguished by their underlying organization of emotion information. Our findings demonstrate that the classic notions of valence and arousal as explanatory dimensions of emotions apply differentially to the subtypes that we identified. That is, although all subtypes' emotion spaces were organized predominantly according to valence, which is consistent with prior theory ${ }^{37}$, arousal played a less decisive role as an organizing principle, and only applied to a subset of the participants. The existence of such subtypes suggests distinct sets of abstract emotion spaces that may reflect differences in processing emotion information at some cognitive level ${ }^{38,39}$. These results provide insight to the ongoing debate regarding emotion-related representations in the domain of cognitive neuroscience ${ }^{40,41}$, and, given that emotion dysregulation has been linked to changes in various cognitive functions for patients with schizophrenia ${ }^{42}$, borderline personality disorder ${ }^{43}$, and major depression ${ }^{44}$, these findings can also offer a new perspective to emotion-related psychiatric disorders.

The importance of investigating emotions at the individual level has, however, been previously addressed ${ }^{45}$. For example, earlier behavioral ${ }^{46}$, physiological ${ }^{47}$, and functional neuroimaging studies ${ }^{48-50}$ examined sex-related differences in emotion processing, while further behavioral ${ }^{51,52}$ and neuroimaging studies ${ }^{53,54}$ sought to correlate emotion processing with other individual traits. Our study differs in that the experimental design was not based on predefined labels (e.g., positive, negative, disgusting, etc.) that could have biased participants while organizing the images (or even constituted potentially irrelevant categories) and would have artificially limited the "resolution" of the emotion space that our analyses could reveal. Thus, the definition of the discrete subtypes was data-driven, as the task was carried out in a non-labeled, continuous space and the analyses were model-free.

However, a few questions emerge from the limitations of our findings. First, despite our resampling simulation, the ability to detect emotion subtypes may be a function of the sample size. That is, using a larger sample increases the accuracy with which one can define subtypes (and their underlying characteristics). For example, two of the subtypes we discovered (i.e., $\alpha$ and $\gamma$ ) were each composed of only one participant. It remains to be seen whether these participants do indeed represent subtypes that simply exist in the population with a lower probability, or whether increasing the sample size would yield a better estimate of each subtype's expected variability, thereby incorporating these two "outlier" participants into other subtypes. Additionally, a larger sample also increases the reliability with which one can find clustering solutions that represent different levels of stability (e.g., the first, second, or nth peak of a silhouette profile). With a sufficiently large sample, it is conceivable that investigating, for example, fourth-level stability could expose meaningful information about subtypes.

Second, it is not clear whether our findings would generalize when using a different stimulus set. Employing images from the IAPS allowed us to ultimately make objective claims regarding the applicability of valence and arousal as meaningful dimensions, but an extended stimulus set, also from other databases or even other modalities (e.g., auditory stimuli, words, etc.), that contained standardized ratings for additional stimulus properties would reinforce the validity of our findings.

Third, the question remains open as to which character- 


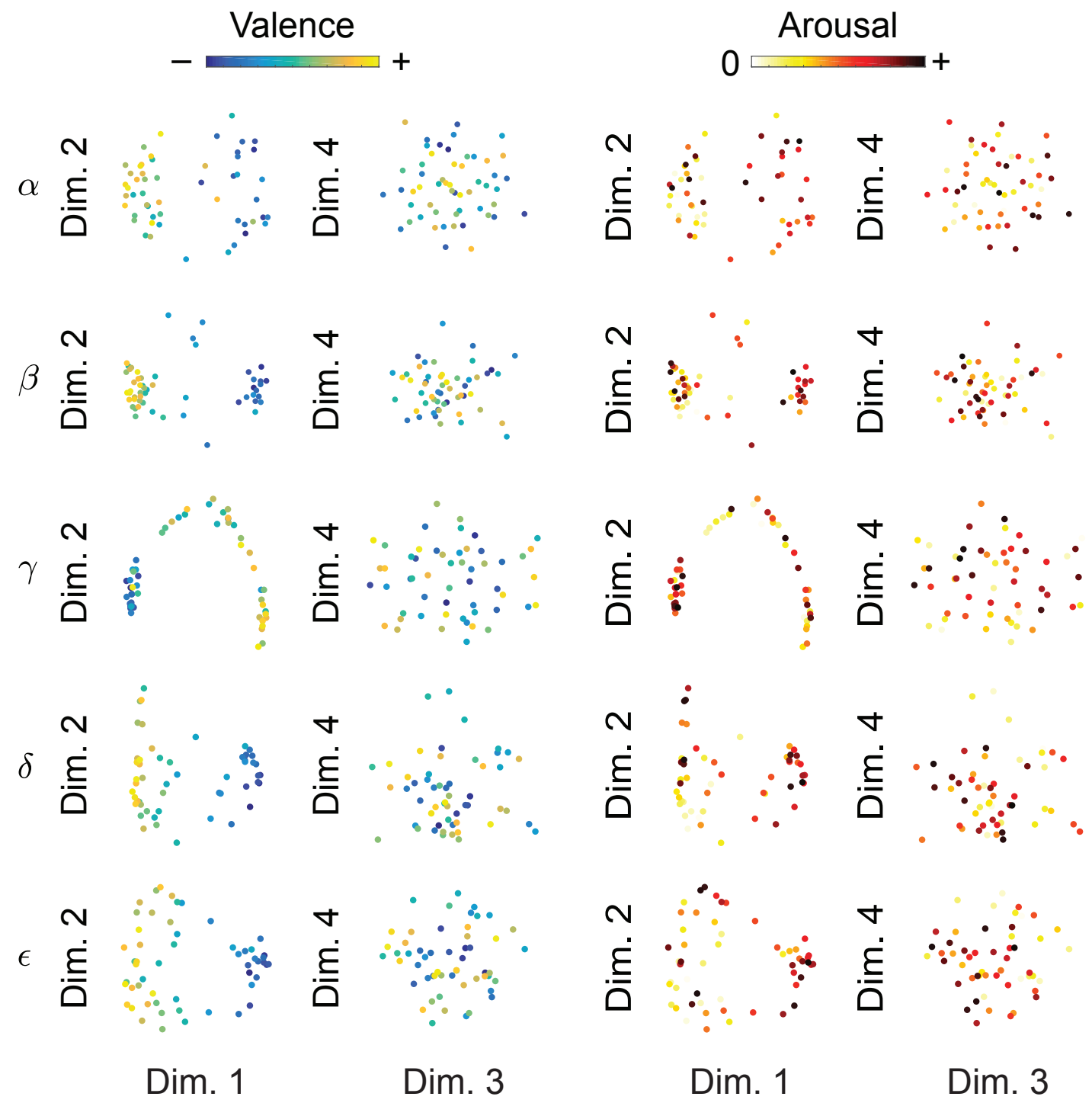

Fig. 4. Averaging the dissimilarity matrices from all participants of each subtype yielded five subtype-representative dissimilarity matrices. To visualize the underlying structure of each subtype's emotion space in terms of the classic dimensions of valence and arousal, we reduced the subtype-representative dissimilarity matrices to four dimensions using non-metric multidimensional scaling and generated respective scatterplots overlaying heatmaps for each image's valence (left column) and arousal (right column) scores. Note how, for all subtypes, valence follows a clear gradient along the first dimension (left-to-right; reversed for some subtypes), whereas the change in arousal along the dimensions is less striking. See Figure 5, Supplementary table 2 and Supplementary table 3 for the respective descriptive statistics.

istics of the subtypes' emotion spaces map onto the underlying dimensions. Although valence was the predominant organizing principle, mapping rather cleanly onto the first dimension for all subtypes, arousal played a much smaller role and for a limited set of subtypes. Furthermore, there are additional dimensions, beyond those that we investigated in the present analyses, necessary to fully reconstruct a given subtype's emotion space. For perspective, reducing subtype delta's DSM to four dimensions yielded a stress value of 0.056, whereas reducing the same DSM to 10 dimensions yielded a stress value of 0.027. Moreover, reducing subtype alpha's DSM to four dimensions yielded a stress value of 0.187 , while 10 dimensions still yielded a stress value of 0.089 These data raise the question of which aspects of the emotion space do these higher dimensions represent.

Lastly, and possibly most crucial for future investigations, remains the questions as to whether the emotion subtypes we identified reflect states or traits. It is possible that the manner in which participants organized the stimuli is a reflection of temporary mental states or transient cognitive functions that were employed during the experimental task rather than more permanent aspects of their personalities. Assessing re-test reliability with the same set of participants, combining such methods with neuroimaging experiments, and sampling from clinical populations would be critical steps in determining the degree to which investigating emotion spaces can accurately uncover features of the cognitive architecture and shed light on characteristics of various psychiatric conditions.

Nevertheless, we feel that the methods and results we present hereinabove demonstrate a crucial new perspective in emotion-related research. The possibility of exploring emotion subtypes as explanatory variables leads to a new set of 


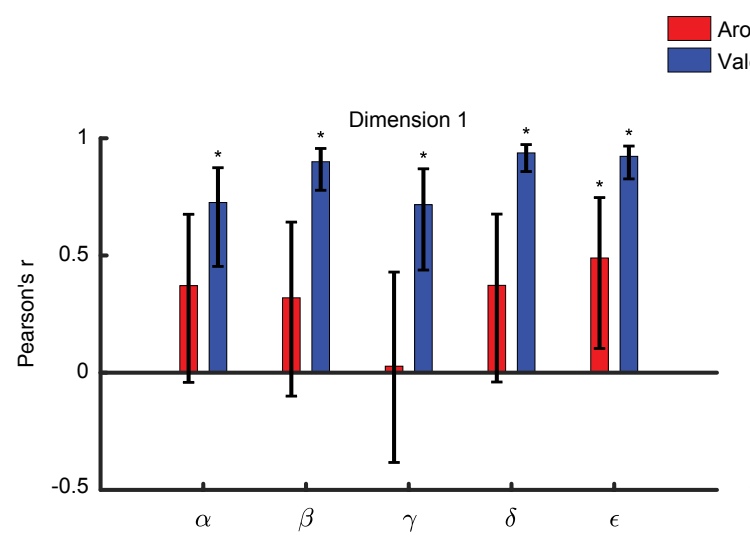

Arousal

Valence
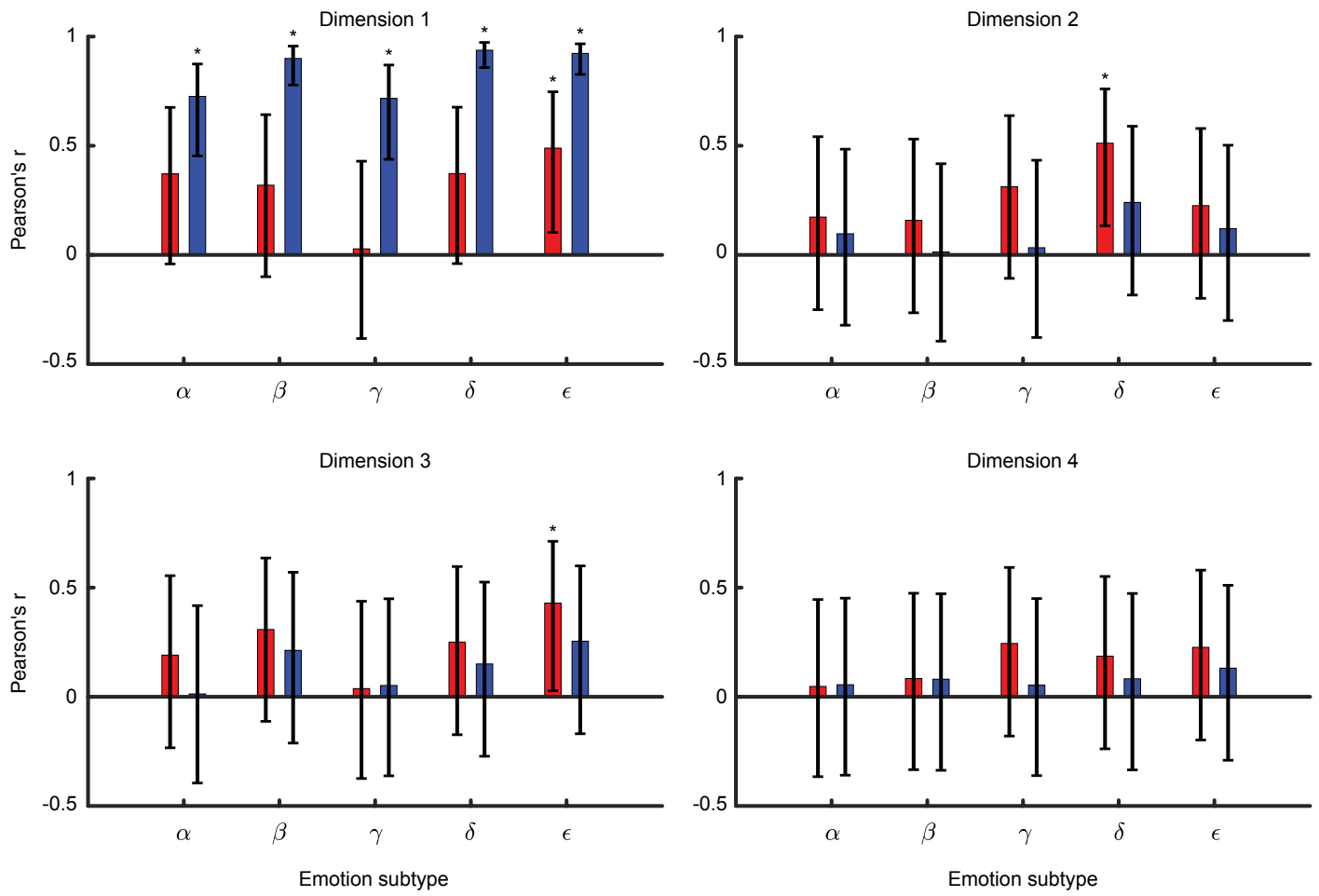

Fig. 5. For each subtype we partial-correlated the valence (blue) and arousal (red) scores from the stimuli with their location along the four dimensions (see Figure 4), which verified that valence plays a strong role in explaining the organization of stimuli along the first dimension for all subtypes. Interestingly, for only two subtypes (i.e., $\delta$ and $\epsilon$ ) did arousal define the arrangement of data in any dimensions and far more limitedly than valence. This observation suggests that classic models of emotion apply differentially in describing emotion information processing for different people. All correlations are presented here as positive for easier visual comparison, since the direction of the effect is not the primary feature of interest. Error bars represent Fisher-transformed FDR-corrected 95\% confidence intervals; asterisks depict statistical tests for which we rejected the null hypothesis of no correlation at the FDR-corrected threshold $(q=0.05, p<0.0014$, two-tailed).

questions for the cognitive and psychological sciences, such as, for example, whether certain emotion subtypes are more at risk for specific psychiatric disorders, whether the complexity (or simplicity) of people's emotion spaces (as measured by the number of underlying dimensions necessary to accurately reconstruct it) corresponds to other aspects of their cognition, or whether changes in emotion space relate to meaningful changes in behavior, physiology, or other cognitive functions. These ideas highlight the importance of focusing on information at the individual level with new computational methods in order for basic research to translate into the medical domain with maximal impact.

\section{Acknowledgments}

The authors would like to thank Angelika Lingnau and Marieke Mur for contributing experimental code for data acquisition and Caroline Nothdurfter for comments on earlier versions of this manuscript.

The formatting style for this manuscript is a derivative of the "HenriquesLab bioRxiv template" by Ricardo Henriques, used under CC BY 4.0.

\section{Conflict of interest}

The authors declare no financial conflicts of interest.

\section{References}

1. Hamann S. Mapping discrete and dimensional emotions onto the brain: Controversies and consensus. Trends Cogn Sci 2012; 16: 458.

2. Lindquist KA, Wager TD, Kober H, Bliss-Moreau E, Barrett LF. The brain basis of emotion: A metaanalytic review. Behav Brain Sci 2012; 35: 121-143.

3. Barrett LF. The theory of constructed emotion: an active inference account of interoception and categorization. Soc Cogn Affect Neurosci 2017; 12: 1-23.

4. Russell JA. A circumplex model of affect. J Pers Soc Psychol 1980; 39: 1161-1178.

5. Barrett LF, Russell JA. The Structure of Current Affect. Curr Dir Psychol Sci 1999; 8: 10-14.

6. Russell JA. Core affect annd the psychological construction of emotion. Psychol Rev 2003; 110: $145-172$.

7. Baucom LB, Wedell DH, Wang J, Blitzer DN, Shinkareva S V. Decoding the neural representation of affective states. Neuroimage 2012; 59: 718-727. 
8. Goodkind MS, Sollberger M, Gyurak A, Rosen HJ, Rankin KP, Miller B et al. Tracking emotional valence: The role of the orbitofrontal cortex. Hum Brain Mapp 2012; 33: 753-762.

9. Nielen MMA, Heslenfeld DJ, Heinen K, Van Strien JW, Witter MP, Jonker C et al. Distinct brain systems underlie the processing of valence and arousal of affective pictures. Brain Cogn 2009; 71: 387-396.

10. Chikazoe J, Lee DH, Kriegeskorte N, Anderson AK. Population coding of affect across stimuli, modalities and individuals. Nat Neurosci 2014; 17: 1114-1122.

11. Chang LJ, Gianaros PJ, Manuck SB, Krishnan A. A Sensitive and Specific Neural Signature for PictureInduced Negative Affect. PLoS Biol 2015; 13: 1-28.

12. Shinkareva S V., Wang J, Kim J, Facciani MJ, Baucom LB, Wedell DH. Representations of modality-specific affective processing for visual and auditory stimuli derived from functional magnetic resonance imaging data. Hum Brain Mapp 2014; 35: 3558-3568.

13. Amstadter $\mathrm{AB}$. Emotion regulation and anxiety disorders. J Anxiety Disord 2008; 22: 211-221.

14. Carpenter RW, Trull TJ. Components of emotion dysregulation in borderline personality disorder: A review. Curr. Psychiatry Rep. 2013; 15: 335.

15. Reimherr FW, Marchant BK, Strong RE, Hedges DW, Adler L, Spencer TJ et al. Emotional dysregulation in adult ADHD and response to atomoxetine. Biol Psychiatry $2005 ; 58: 125-131$.

16. Etkin A, Wager TD. Functional Neuroimaging of Anxiety: A Meta-Analysis of Emotional Processing in PTSD, Social Anxiety Disorder, and Specific Phobia. Am J Psychiatry 2007; 164: 1476-1488.

17. Mennin DS, Heimberg RG, Turk CL, Fresco DM. Preliminary evidence for an emotion dysregulation model of generalized anxiety disorder. Behav Res Ther 2005; 43: $1281-1310$.

18. Taylor SF, Kang J, Brege IS, Tso IF, Hosanagar A, Johnson TD. Meta-analysis of functional neuroimaging studies of emotion perception and experience in schizophrenia. Biol Psychiatry 2012; 71: 136-145.

19. Phillips ML, Drevets WC, Rauch SL, Lane R. Neurobiology of Emotion Perception II: Implications for Major Psychiatric Disorders. Biol Psychiatry 2003; 54: 515528.

20. Hamburg MA, Collins FS. The Path to Personalized Medicine - Perspective. N Engl J Med 2010; 363: 301-304.

21. Friston KJ, Stephan KE, Montague R, Dolan RJ. Computational psychiatry: The brain as a phantastic organ. The Lancet Psychiatry. 2014; 1: 148-158.
22. Huys QJM, Maia T V, Frank MJ. Computational psychiatry as a bridge from neuroscience to clinical applications. Nat Neurosci 2016; 19: 404-413.

23. Cacioppo JT, Cacioppo S, Dulawa S, Palmer AA. Social neuroscience and its potential contribution to psychiatry. World Psychiatry. 2014; 13: 131-139.

24. Goldstone R. An efficient method for obtaining similarity data. Behav Res Methods, Instruments, Comput 1994; 26: 381-386.

25. Kriegeskorte N, Mur M. Inverse MDS: Inferring dissimilarity structure from multiple item arrangements. Front Psychol 2012; 3: 1-13.

26. Mur M, Meys M, Bodurka J, Goebel R, Bandettini PA, Kriegeskorte N. Human object-similarity judgments reflect and transcend the primate-IT object representation. Front Psychol 2013; 4. doi:10.3389/fpsyg.2013.00128.

27. Charest I, Kievit RA, Schmitz TW, Deca D, Kriegeskorte N. Unique semantic space in the brain of each beholder predicts perceived similarity. Proc Natl Acad Sci 2014; 111: 14565-14570.

28. Bracci S, Op de Beeck H. Dissociations and Associations between Shape and Category Representations in the Two Visual Pathways. J Neurosci 2016; 36: 432-444.

29. Bracci S, Caramazza A, Peelen M V. Representational Similarity of Body Parts in Human Occipitotemporal Cortex. J Neurosci 2015; 35: 12977-12985.

30. Lang PJ, Bradley MM, Cuthbert BN. International affective picture system (IAPS): Affective ratings of pictures and instruction manual. Tech Rep A-8 2008.

31. Ward JH. Hierarchical Grouping to Optimize an Objective Function. J Am Stat Assoc 1963; 58: 236-244.

32. Rousseeuw PJ. Silhouettes: A graphical aid to the interpretation and validation of cluster analysis. J Comput Appl Math 1987; 20: 53-65.

33. Stelzer J, Chen Y, Turner R. Statistical inference and multiple testing correction in classification-based multi-voxel pattern analysis (MVPA): Random permutations and cluster size control. Neuroimage 2013; 65: 69-82.

34. Kruskal JB. Multidimensional scaling by optimizing goodness of fit to a nonmetric hypothesis. Psychometrika 1964; 29: 1-27.

35. Benjamini Y, Hochberg Y. Benjamini Y, Hochberg Y. Controlling the false discovery rate: a practical and powerful approach to multiple testing. J R Stat Soc B 1995; 57: 289-300. 
36. Yeo BTT, Krienen FM, Sepulcre J, Sabuncu MR, Lashkari D, Hollinshead $\mathrm{M}$ et al. The organization of the human cerebral cortex estimated by intrinsic functional connectivity. J Neurophysiol 2011; 106: 1125-1165.

37. Barrett LF. Valence is a basic building block of emotional life. J. Res. Pers. 2006; 40: 35-55.

38. Adolphs R. How should neuroscience study emotions? by distinguishing emotion states, concepts, and experiences. Soc Cogn Affect Neurosci 2017; 12: 24-31.

39. Marr D. Vision: A Computational Investigation into the Human Representation and Processing of Visual Information. W.H. Freeman: San Francisco, 1982.

40. Kragel PA, LaBar KS. Decoding the Nature of Emotion in the Brain. Trends Cogn Sci 2016; 20: 444-455.

41. Lindquist KA, Satpute AB, Wager TD, Weber J, Barrett LF. The Brain Basis of Positive and Negative Affect: Evidence from a Meta-Analysis of the Human Neuroimaging Literature. Cereb Cortex 2016; 26: 1910-1922.

42. Gooding DC, Tallent KA. Schizophrenia patients' perceptual biases in response to positively and negatively valenced emotion chimeras. Psychol Med 2002; 32: S0033291702005792.

43. Dyck M, Habel U, Slodczyk J, Schlummer J, Backes $\mathrm{V}$, Schneider F et al. Negative bias in fast emotion discrimination in borderline personality disorder. Psychol Med 2009; 39: 855.

44. Murphy FC, Michael A, Sahakian BJ. Emotion modulates cognitive flexibility in patients with major depression. Psychol Med 2012; 42: 1373-1382.

45. Hamann S, Canli T. Individual differences in emotion processing. Curr. Opin. Neurobiol. 2004; 14: 233-238.

46. Cahill L, Gorski L, Belcher A, Huynh Q. The influence of sex versus sex-related traits on long-term memory for gist and detail from an emotional story. Conscious Cogn 2004; 13: 391-400.

47. Bradley MM, Codispoti M, Sabatinelli D, Lang PJ. Emotion and motivation II: sex differences in picture processing. Emotion 2001; 1: 300-319.

48. Koch K, Pauly K, Kellermann T, Seiferth NY, Reske $\mathrm{M}$, Backes V et al. Gender differences in the cognitive control of emotion: An fMRI study. Neuropsychologia 2007; 45: 2744-2754.

49. McRae K, Ochsner KN, Mauss I, Gabrieli J. Gender differences in emotion regulation: An fMRI study of cognitive reappraisal. Gr Process Intergr Relations 2008; 11: 143-162.
50. Schienle A, Schafer A, Stark R, Walter B, Vaitl D. Gender differences in the processing of disgust- and fearinducing pictures: an fMRI study. Neuroreport 2005; 16: $277-280$.

51. Waring JD, Payne JD, Schacter DL, Kensinger EA. Impact of individual differences upon emotion-induced memory trade-offs. Cogn. Emot. 2010; 24: 150-167.

52. Mueller CJ, Kuchinke L. Individual differences in emotion word processing: A diffusion model analysis. Cogn. Affect. Behav. Neurosci. 2016; 16: 489-501.

53. Mériau K, Wartenburger I, Kazzer P, Prehn K, Lammers $\mathrm{CH}$, van der Meer E et al. A neural network reflecting individual differences in cognitive processing of emotions during perceptual decision making. Neuroimage 2006; 33: 1016-1027.

54. Lee H, Heller AS, van Reekum CM, Nelson B, Davidson RJ. Amygdala-prefrontal coupling underlies individual differences in emotion regulation. Neuroimage 2012; 62: 1575-1581. 


\section{Appendix}

\begin{tabular}{|c|c|c|c|}
\hline Stimulus ID & Description & Valence rating & Arousal rating \\
\hline 1030 & Snake & 4.3 & 5.46 \\
\hline 1202 & Spider & 3.35 & 5.94 \\
\hline 1333 & Parrot & 6.11 & 3.17 \\
\hline 1640 & Coyote & 6.27 & 5.13 \\
\hline 1670 & Cow & 6.81 & 3.05 \\
\hline 1903 & Shrimp & 5.5 & 4.25 \\
\hline 2102 & Man Sitting & 5.16 & 3.03 \\
\hline 2260 & Baby & 8.06 & 4.26 \\
\hline 2300 & Lady in a Dress & 7.04 & 5.55 \\
\hline 2441 & Staring Girl & 4.64 & 3.62 \\
\hline 2593 & Cafe & 5.8 & 3.42 \\
\hline 2900.2 & Happy Girl & 6.62 & 4.52 \\
\hline 3051 & Beaten Woman & 2.3 & 5.62 \\
\hline 3210 & Surgery & 4.49 & 5.39 \\
\hline 3310 & Preemie & 4.37 & 5.43 \\
\hline 3360 & Fetus & 3.78 & 5.39 \\
\hline 3400 & Severed Hand & 2.35 & 6.91 \\
\hline 3550 & Beaten Man & 2.54 & 5.92 \\
\hline 4085 & Erotic Female & 5.71 & 5.77 \\
\hline 4250 & Attractive Female & 6.79 & 5.16 \\
\hline 4525 & Attractive Male & 6.51 & 5.17 \\
\hline 4660 & Erotic Couple 1 & 7.4 & 6.58 \\
\hline 4698 & Erotic Couple 2 & 6.5 & 6.72 \\
\hline 4700 & Happy Couple & 6.91 & 4.05 \\
\hline 5030 & Flower 1 & 6.51 & 2.74 \\
\hline 5202 & Flower 2 & 7.25 & 3.73 \\
\hline 5622 & Shark & 6.33 & 5.34 \\
\hline 5780 & Lake & 7.52 & 3.75 \\
\hline 5831 & Seagulls at the Beach & 7.63 & 4.43 \\
\hline 5982 & Clouds & 7.61 & 4.51 \\
\hline 6020 & Execution Chair & 3.41 & 5.58 \\
\hline 6200 & Aimed Gun 1 & 2.71 & 6.21 \\
\hline 6241 & Gun & 3.42 & 4.54 \\
\hline 6250 & Aimed Gun 2 & 2.83 & 6.54 \\
\hline 6832 & Aimed Gun 3 & 4.02 & 5.51 \\
\hline 6940 & Tank & 3.53 & 5.35 \\
\hline 7002 & Towel & 4.97 & 3.16 \\
\hline 7039 & Train & 5.93 & 3.29 \\
\hline 7042 & Hand Weights & 5.55 & 4.02 \\
\hline 7405 & Cupcakes & 7.38 & 6.28 \\
\hline 7512 & Chess & 5.38 & 3.72 \\
\hline 7521 & Hospital Bed & 3.92 & 4.38 \\
\hline 8120 & Sporty Girl & 7.09 & 4.85 \\
\hline 8163 & Parachutist & 7.14 & 6.53 \\
\hline 8200 & Water Skier & 7.54 & 6.35 \\
\hline 8211 & Boat & 5.76 & 5.36 \\
\hline 8370 & Rafting & 7.77 & 6.73 \\
\hline 8380 & Medalists & 7.56 & 5.74 \\
\hline 9185 & Dead Dog & 1.97 & 5.65 \\
\hline 9420 & Dead Man & 2.31 & 5.69 \\
\hline 9422 & Battle Ship & 4.95 & 5.09 \\
\hline 9495 & Revolutionists & 3.34 & 5.57 \\
\hline 9622 & Exploding Jet in Sky & 3.1 & 6.26 \\
\hline 9908 & Car Accident & 2.34 & 6.63 \\
\hline
\end{tabular}

Supplementary table 1. Descriptive information for each of the images from the International Affective Picture System that we used as stimuli in the experiment. Because of spatial constraints, the stimulus descriptions were omitted from the figures (e.g., next to the dissimilarity matrices where they would otherwise be illegible), but the order of images presented in the table is the same as the order of images represented by each row of the $54 \times 54$ dissimilarity matrices. 


\begin{tabular}{|c|c|c|c|c|c|c|c|c|}
\cline { 2 - 9 } \multicolumn{1}{c|}{} & \multicolumn{9}{c|}{ Valence } \\
\cline { 2 - 9 } \multicolumn{1}{c|}{} & \multicolumn{2}{c|}{ Dimension 1 } & \multicolumn{2}{c|}{ Dimension 2 } & \multicolumn{2}{c|}{ Dimension 3 } & \multicolumn{2}{c|}{ Dimension 4 } \\
\hline Subtype & $\mathrm{r}$ & $\mathrm{p}$ & $\mathrm{r}$ & $\mathrm{p}$ & $\mathrm{r}$ & $\mathrm{p}$ & $\mathrm{r}$ & $\mathrm{p}$ \\
\hline$\alpha$ & -0.73 & $\mathbf{7 . 7} \times \mathbf{1 0}^{-10}$ & 0.10 & 0.49 & -0.01 & 0.93 & -0.05 & 0.70 \\
\hline$\beta$ & -0.90 & $\mathbf{5 . 1} \times \mathbf{1 0}^{-20}$ & -0.01 & 0.92 & -0.21 & 0.13 & -0.08 & 0.57 \\
\hline$\gamma$ & 0.72 & $\mathbf{1 . 6} \times \mathbf{1 0}^{-9}$ & 0.03 & 0.81 & -0.05 & 0.72 & 0.05 & 0.71 \\
\hline$\delta$ & -0.94 & $\mathbf{4 . 9} \times \mathbf{1 0}^{-25}$ & 0.24 & 0.08 & -0.15 & 0.29 & -0.08 & 0.56 \\
\hline$\epsilon$ & -0.92 & $\mathbf{8 . 4} \times \mathbf{1 0}^{-23}$ & 0.12 & 0.39 & -0.25 & 0.07 & -0.13 & 0.35 \\
\hline
\end{tabular}

Supplementary table 2. Descriptive statistics underlying Figure 5 for the partial correlation (Pearson's $r$ ) between images' valence values and their organization along the four dimensions for each emotion subtype. P-values that were smaller than the FDR correction $(q=0.05$, two-tailed) are presented in bold.

\begin{tabular}{|c|c|c|c|c|c|c|c|c|}
\cline { 2 - 9 } \multicolumn{1}{c|}{} & \multicolumn{9}{c|}{ Arousal } \\
\cline { 2 - 9 } \multicolumn{1}{c|}{} & \multicolumn{2}{c|}{ Dimension 1 } & \multicolumn{2}{c|}{ Dimension 2 } & \multicolumn{2}{c|}{ Dimension 3 } & \multicolumn{2}{c|}{ Dimension 4 } \\
\hline Subtype & $\mathrm{r}$ & $\mathrm{p}$ & $\mathrm{r}$ & $\mathrm{p}$ & $\mathrm{r}$ & $\mathrm{p}$ & $\mathrm{r}$ & $\mathrm{p}$ \\
\hline$\alpha$ & 0.37 & $6.2 \times 10^{-3}$ & 0.17 & 0.22 & 0.19 & 0.17 & -0.05 & 0.74 \\
\hline$\beta$ & 0.32 & 0.02 & 0.16 & 0.26 & -0.31 & 0.03 & 0.08 & 0.56 \\
\hline$\gamma$ & -0.03 & 0.84 & -0.31 & 0.02 & -0.04 & 0.79 & 0.24 & 0.08 \\
\hline$\delta$ & 0.37 & $6.2 \times 10^{-3}$ & 0.51 & $\mathbf{8 . 9} \times \mathbf{1 0}^{-5}$ & -0.25 & 0.07 & -0.18 & 0.19 \\
\hline$\epsilon$ & 0.49 & $\mathbf{2 . 0} \times \mathbf{1 0}^{-4}$ & 0.23 & 0.10 & -0.43 & $\mathbf{1 . 4}^{-4} \times \mathbf{1 0}^{-3}$ & -0.23 & 0.11 \\
\hline
\end{tabular}

Supplementary table 3. Descriptive statistics underlying Figure 5 for the partial correlation (Pearson's $r$ ) between images' arousal values and their organization along the four dimensions for each emotion subtype. P-values that were smaller than the FDR correction $(q=0.05$, two-tailed) are presented in bold. 